\title{
On the Cyclic Homology of Hopf Crossed Products
}

\author{
M. Khalkhali, and B. Rangipour, \\ masoud@uwo.ca brangipo@uwo.ca \\ Department of Mathematics \\ University of Western Ontario \\ London, ON, Canada
}

\begin{abstract}
We consider Hopf crossed products of the the type $A \#_{\sigma} \mathcal{H}$, where $\mathcal{H}$ is a cocommutative Hopf algebra, $A$ is an $\mathcal{H}$-module algebra and $\sigma$ is a "numerical" convolution invertible 2 -cocycle on $\mathcal{H}$. we give an spectral sequence that converges to the cyclic homology of $A \#{ }_{\sigma} \mathcal{H}$ and identify the $E^{1}$ and $E^{2}$ terms of the spectral sequence.
\end{abstract}

\section{Introduction}

A celebrated problem in noncommutative geometry, more precisely in cyclic homology theory, is to compute the cyclic homology of a crossed product algebra. The interest in this problem stems from the fact that, according to a guiding principle in noncommutative geometry [3], crossed products play the role of "noncommutative quotients" in situations where the usual set theoretic quotients are ill behaved. For example, when a (locally compact) group $G$ acts on a locally compact Hausdorff space $X$, the quotient space $X / G$ may not be well behaved, e.g. may not be even a Hausdorff space. The crossed product $\left(C^{*}-\right)$ algebra $C_{0}(X) \ltimes G$, however, is a good replacement for $X / G[3$. In fact, if the action of $G$ is free and proper, then by a theorem of Rieffel [13] the $C^{*}$-algebra of continuous functions vanishing at infinity on $X / G$, denoted by $C_{0}(X / G)$ is in a suitable $C^{*}$-algebraic sense, Morita equivalent to the crossed product algebra $C_{0}(X) \ltimes G$. Since $K$-Theory, Hochschild 
homology and cyclic homology are Morita invariant functors, replacing the commutative algebra $C_{0}(X / G)$ by the noncommutative algebra $C_{0}(X) \ltimes G$ results in no loss of information.

It is therefore natural and desirable to develop tools to compute the cyclic homology of crossed product algebras. Most of the results obtained so far are concerned with the action of groups on algebras [6, 7, 12. For Hopf algebra crossed products, 8] gives a complete answer for Hochschild homology but it is not clear how to extend its method to cyclic homology. If one is only interested in smash products as opposed to crossed products, one can find a complete answer in [1] in terms of a spectral sequence converging to the cyclic homology of the smash product algebra $A \# \mathcal{H}$.

The goal of this article is to extend the results of [1] to Hopf algebra crossed products. Due to the fact that very complicated formulas appear in our constructions, however, we had to assume that the Hopf algebra is cocommutative and the 2-cocycle takes values in the ground field. Under these conditions, we give a spectral sequence for the cyclic homology of a crossed product algebra $\underset{\sigma}{A \# \mathcal{H}}$, when the cocycle $\sigma$ is convolution invertible and takes values in the ground field $k$. The method of proof is similar to the one used in [1] which is based on the generalized cyclic Eilenberg-Zilber theorem of [7]. Though we think the same method should apply to arbitrary crossed products $A \underset{\sigma}{A} \mathcal{H}$ (with convolution invertible cocycles), due to technical difficulties we are not able to verify this.

\section{Preliminaries}

In this paper we work over a fixed ground field $k$. All algebras are unital associative algebras over $k$ and all modules are unitary. The unadorned tensor product $\otimes$ means tensor product over $k$. We denote the coproduct of a Hopf algebra by $\Delta$, the counit by $\epsilon$ and the antipode by $S$. We use Sweedler's notation and write $\Delta(h)=h^{(1)} \otimes h^{(2)}$ to denote the coproduct, where summation is understood. Similarly, we write $\Delta^{(n)}(h)=h^{(1)} \otimes h^{(2)} \otimes$

$\ldots \otimes h^{(n+1)}$ to denote the iterated coproducts defined by $\Delta^{(1)}=\Delta$ and $\Delta^{(n+1)}=(\Delta \otimes 1) \circ \Delta^{(n)}$.

Let $\mathcal{H}$ be a Hopf algebra and $A$ an algebra. Recall from [2] and [5] that a weak action of $\mathcal{H}$ on $A$ is a linear map $\mathcal{H} \otimes A \longrightarrow A, h \otimes a \rightarrow h(a)$ such that, for all $h \in \mathcal{H}$, and $a, b \in A$ 
1) $h(a b)=h^{(1)}(a) h^{(2)}(b)$,

2) $\quad h(1)=\epsilon(h) 1$

3) $1(a)=a$.

By an action of $\mathcal{H}$ on $A$, we mean a week action such that $A$ is an $\mathcal{H}$ module, i.e. for all $h, l \in \mathcal{H}$ and $a \in A$ we have $h(l(a))=h l(a)$. In the latter case we say $A$ is an $\mathcal{H}$-module algebra.

Let $A$ be an $\mathcal{H}$-module algebra. The smash product $A \# \mathcal{H}$ of $A$ and $\mathcal{H}$ is an associative algebra whose underlying vector space is $A \otimes \mathcal{H}$ and whose multiplication is defined by

$$
(a \otimes h)(b \otimes l)=a h^{(1)}(b) \otimes h^{(2)} l .
$$

If, on the other hand, we have only a week action of $\mathcal{H}$ on $A$ the above formula does not define an associative multiplication, and a modification is needed. Given a linear map $\sigma: \mathcal{H} \otimes \mathcal{H} \rightarrow A$ one defines a (not necessarily unital or associative) multiplication on $A \otimes \mathcal{H}$ by [2, 5]

$$
(a \otimes h)(b \otimes l)=a h^{(1)}(b) \sigma\left(h^{(2)}, l^{(1)}\right) \otimes h^{(3)} l^{(2)} .
$$

It can be shown that the above formula defines an associative product with $1 \otimes 1$ as its unit, if and only if $\sigma$ and the weak action enjoy the following properties:

1) (Normality) For all $h \in \mathcal{H}, \sigma(h, 1)=\sigma(1, h)=\epsilon(h) 1$.

2) (Cocycle property) For all $h, l, m \in \mathcal{H}$,

$$
\sum h^{(1)}\left(\sigma\left(l^{(1)}, m^{(1)}\right)\right) \sigma\left(h^{(2)}, l^{(2)} m^{(2)}\right)=\sum \sigma\left(h^{(1)}, l^{(1)}\right) \sigma\left(h^{(2)} l^{(2)}, m\right),
$$

3) (Twisted module property) For all $h, l \in \mathcal{H}$ and $a \in A$, $\left.\sum h^{(1)}\left(l^{(1)}(a)\right) \sigma\left(h^{(2)}, l^{(2)}\right)=\sum \sigma\left(h^{(1)}, l^{(1)}\right) l^{(2)} h^{(2)}(a)\right)$.

The cocycle $\sigma$ is said to be convolution invertible if it is an invertible element of the convolution algebra $\operatorname{Hom}_{k}(\mathcal{H} \otimes \mathcal{H}, A)$. Now assume the Hopf algebra $\mathcal{H}$ is cocommutative, $\sigma: \mathcal{H} \otimes \mathcal{H} \longrightarrow k 1_{A}$ takes values in the ground field $k$, and $\sigma$ is invertible. Then it follows that $\mathrm{A}$ is an $\mathcal{H}$-module algebra, i.e. the weak action in the above situation is in fact an action. To prove this let $a \in A$ be fixed. Define two functions in $H_{o m}(\mathcal{H} \otimes \mathcal{H}, A)$ by $F(h, l)=$ 
$\sum h^{(1)}\left(l^{(1)}(a)\right) \sigma\left(h^{(2)}, l^{(2)}\right)$ and $G(h, l)=\sum \sigma\left(h^{(1)}, l^{(1)}\right) l^{(2)} h^{(2)}(a)$. Then $F=G$ by the twisted module property of $\sigma$, so $F * \sigma^{-1}=G * \sigma^{-1}$. In other word

$$
h(l(a))=F * \sigma^{-1}(h, l)=G * \sigma^{-1}(h, l)=h l(a) .
$$

One notes that the above proof remains valid when $\mathcal{H}$ is cocommutative and $\sigma$ takes its values in the center of $A$, instead of $k$.

One of the main technical tools used in [1] to derive a spectral sequence for the cyclic homology of smash products is the generalized cyclic EilenbergZilber theorem. This result was first stated in [7] but its first algebraic proof appeared in [10]. The idea of using an Eilenberg-Zilber type theorem to derive a spectral sequence for cyclic homology of smash products (for the action of groups) is due to Getzler and Jones [7. We find it remarkable that the same idea works in the case of Hopf algebra crossed product (with convolution invertible cocycle). In the following we recall the definitions of (para)cyclic modules, cylindrical modules and state the Eilenberg-Zilber theorem for cylindrical modules.

Recall that a paracyclic module is a simplicial $k$-module $\left\{M_{n}\right\}_{n \geq 0}$, such that the following extra relations are satisfied [6, 7]:

$$
\begin{array}{lll}
\delta_{i} \tau=\tau \delta_{i-1}, & \delta_{0} \tau=\delta_{n}, & 1 \leq i \leq n, \\
\sigma_{i} \tau=\tau \sigma_{i+1}, & \sigma_{0} \tau=\tau^{2} \sigma_{n}, & 1 \leq i \leq n,
\end{array}
$$

where $\delta_{i}: M_{n} \rightarrow M_{n-1}, \quad \sigma_{i}: M_{n} \rightarrow M_{n+1}, 0 \leq i \leq n$, are faces and degeneracies of the simplicial module $\left\{M_{n}\right\}_{n \geq 0}$ and $\tau: M_{n} \rightarrow M_{n}, n \geq 0$ are $k$-linear maps. If furthermore we have $\tau^{n+1}=i d_{M_{n}}$, for all $n \geq 0$, then we say that we have a cyclic module.

We denote the cyclic module of an associative unital $k$-algebra $A$, by $A^{\natural}$. It is defined by $A_{n}^{\natural}=A^{\otimes(n+1)}, n \geq 0$, and simplicial and cyclic operations defined by

$$
\begin{aligned}
\delta_{i}\left(a_{0} \otimes \cdots \otimes a_{n}\right) & =a_{0} \otimes a_{1} \otimes \cdots \otimes a_{i} a_{i+1} \cdots \otimes a_{n}, \quad 0 \leq i \leq n-1, \\
\delta_{n}\left(a_{0} \otimes \cdots \otimes a_{n}\right) & =a_{n} a_{0} \otimes a_{1} \otimes \cdots \otimes a_{n-1}, \\
\sigma_{i}\left(a_{0} \otimes \cdots \otimes a_{n}\right) & =a_{0} \otimes \cdots \otimes a_{i} \otimes 1 \cdots \otimes a_{n}, \quad 0 \leq i \leq n, \\
\tau_{n}\left(a_{0} \otimes \cdots \otimes a_{n}\right) & =a_{n} \otimes a_{0} \cdots \otimes a_{n-1} .
\end{aligned}
$$

To any cyclic module one associates its cyclic homology groups [11, 4]. In particular, the cyclic homology groups of $A^{\natural}$ are devoted by $H C_{n}(A), n \geq 0$, and are called cyclic homology of $A$. 
By a biparacyclic module we mean a doubly graded sequence of $k$-modules $\left\{M_{p, q}\right\}_{p, q \geq 0}$ such that each row and each column is a paracyclic module and all vertical operators commute with all horizontal operators. In particular a bicyclic module is a biparacyclic module such that each row and each column is a cyclic module. We denote the horizontal and vertical operators of a biparacyclic module by $\left(\delta_{i}, \sigma_{i}, \tau\right)$ and $\left(d_{i}, s_{i}, t\right)$ respectively. By a cylindrical module we mean a biparacyclic module such that for all $p, q \geq 0$,

$$
\tau^{p+1} t^{q+1}=i d_{M_{p, q}} .
$$

Given a cylindrical module $M$, its diagonal, denoted by $d M$, is a cyclic module defined by $(d M)_{n}=M_{n, n}$ and with simplicial and cyclic operators given by $\delta_{i} d_{i}, \sigma_{i} s_{i}$, and $\tau t$. In view of (1), it is a cyclic module. The total complex of a cylindrical module, denoted by $\operatorname{Tot}(M)$, is a mixed complex with operators given by $b+\bar{b}$ and $B+T \bar{B}$, where $T=1-(b B+B b)$. Here $b$ (resp. $\bar{b}$ ) and $B$ (resp. $\bar{B}$ ) are the vertical (resp. horizontal) Hochschild and Connes boundary operators of cyclic modules. Note that it differs from the usual notion of total complex in that we use $B+T \bar{B}$ instead of $B+\bar{B}$. In fact the latter choice won't give us a mixed complex [7]. It can be checked that $\operatorname{Tot}(M)$ is a mixed complex. Given a cylindrical or cyclic module $M$, we denote its normalization by $N(M)$.

The following theorem is the main technical result that enables us to derive spectral sequences for the cyclic homology of crossed product algebras.

Theorem 2.1. (Generalized cyclic Eilenberg-Zilber theorem) ([10, 7])For any cylindrical module $M$ there is a natural quasi-isomorphism of mixed complexes $f_{0}+u f_{1}: \operatorname{Tot}(N(M)) \longrightarrow N(d M)$, where $f_{0}$ is the shuffle map.

\section{A Spectral sequence for Hopf crossed prod- ucts}

Let $\mathcal{H}$ be a cocommutative Hopf algebra, $A$ a left $\mathcal{H}$ - module algebra and $\sigma: \mathcal{H} \otimes \mathcal{H} \longrightarrow k 1_{A}$ a two cocycle satisfying the cocycle conditions 1$), 2$ ), and 3) in Section 2. We further assume that $\sigma$ is convolution invertible. We introduce a cylindrical module

$$
A \natural_{\sigma} \mathcal{H}=\left\{\mathcal{H}^{\otimes(p+1)} \otimes A^{\otimes(q+1)}\right\}_{p, q \geq 0}
$$


with vertical and horizontal simplicial and cyclic operators $(\delta, \sigma, \tau)$ and $(d, s, t)$, defined as follows

$$
\begin{aligned}
& \tau\left(g_{0}, \ldots, g_{p} \mid a_{0}, \ldots, a_{q}\right)=\left(g_{0}^{(2)}, \ldots, g_{p}^{(2)} \mid\right. \\
& \left.S\left(g_{0}^{(1)} \ldots g_{p}^{(2)}\right)\left(a_{q}\right), a_{0}, a_{1}, \ldots, a_{q-1}\right) \\
& \delta_{i}\left(g_{0}, \ldots, g_{p} \mid a_{0}, \ldots, a_{q}\right)=\left(g_{0}, \ldots, g_{p} \mid a_{0}, \ldots, a_{i} a_{i+1}, \ldots, a_{q}\right) \quad 0 \leq i<q \\
& \delta_{q}^{p, q}\left(g_{0}, \ldots, g_{p} \mid a_{0}, \ldots, a_{q}\right)=\left(g_{0}^{(2)}, \ldots, g_{p}^{(2)} \mid\right. \\
& \left.S\left(g_{0}^{(1)} \ldots g_{p}^{(2)}\right)\left(a_{q}\right) a_{0}, a_{1} \ldots, a_{q-1}\right) \\
& \sigma_{i}\left(g_{0}, \ldots, g_{p} \mid a_{0}, \ldots, a_{q}\right)=\left(g_{0}, \ldots, g_{p} \mid a_{0}, \ldots, a_{i}, 1, a_{i+1}, \ldots, a_{q}\right) 0 \leq i \leq q \\
& t\left(g_{0}, \ldots, g_{p} \mid a_{0}, \ldots, a_{q}\right)=\left(g_{p}^{(q+2)}, g_{0}, \ldots, g_{p-1} \mid g_{p}^{(1)}\left(a_{0}\right), \ldots, g_{p}^{(q+1)}\left(a_{q}\right)\right) \\
& d_{i}\left(g_{0}, \ldots, g_{p} \mid a_{0}, \ldots, a_{q}\right)=\left(g_{0}, \ldots, g_{i}^{(1)} g_{i+1}^{(1)}, \ldots, g_{p} \mid\right. \\
& \left.\sigma\left(g_{i}^{(2)}, g_{i+1}^{(2)}\right) a_{0}, \ldots, a_{q}\right) \quad 0 \leq i<p \\
& d_{p}\left(g_{0}, \ldots, g_{p} \mid a_{0}, \ldots, a_{q}\right)=\left(g_{p}^{(q+2)} g_{0}^{(1)}, g_{1}, \ldots, g_{p-1} \mid\right. \\
& \left.\sigma\left(g_{p}^{(q+3)}, g_{0}^{(2)}\right) g_{p}^{(1)}\left(a_{0}\right), \ldots, g_{p}^{(q+1)}\left(a_{q}\right)\right) \\
& s_{i}\left(g_{0}, \ldots, g_{p} \mid a_{0}, \ldots, a_{q}\right)=\left(g_{0}, \ldots, g_{i}, 1, g_{i+1}, \ldots, g_{p} \mid a_{0}, \ldots, a_{q}\right) \quad 0 \leq i \leq p .
\end{aligned}
$$

Theorem 3.1. Endowed with the above operators, $A \natural_{\sigma} \mathcal{H}$ is a cylindrical module.

Proof. We should check that every row and every column is a paracyclic module, and vertical operator commutes with each horizontal operator, and in addition the identity (11) holds. Since the weak action in our situation is actually an action and the vertical operators are the same as the vertical operators in (1] Theorem 3.1), we refer the reader to [1] for the proof that the columns form paracyclic modules. To check that the rows are paracyclic modules we need to verify the following identities

$$
\begin{aligned}
d_{i} d_{j} & =d_{j-1} d_{i} \quad i<j \\
s_{i} s_{j} & =s_{j+1} s_{i} \quad i \leq j \\
d_{i} s_{j} & = \begin{cases}s_{j-1} d_{i} \quad i<j \\
\text { identity } & i=j \text { or } i=j+1 \\
s_{j} d_{i-1} & i>j+1 .\end{cases}
\end{aligned}
$$




$$
\begin{array}{r}
d_{i} t_{n}=t_{n-1} d_{i-1} \quad 1 \leq i \leq n, \quad d_{0} t_{n}=d_{n} \\
s_{i} t_{n}=t_{n+1} s_{i-1} \quad 1 \leq i \leq n, \quad s_{0} t_{n}=t_{n+1}^{2} s_{n}
\end{array}
$$

We just check $d_{i} d_{i+1}=d_{i} d_{i}$ and the cylindrical module condition (11). The rest can be proved by the same techniques. We have:

$$
\begin{aligned}
& d_{i} d_{i+1}\left(g_{0}, \ldots, g_{p} \mid a_{0}, \ldots, a_{q}\right)= \\
& d_{i}\left(g_{0}, \ldots, g_{i+1}^{(1)} g_{i+2}^{(1)}, \ldots g_{p} \mid \sigma\left(g_{i+1}^{(2)}, g_{i+2}^{(2)}\right) a_{0}, \ldots, a_{q}\right)= \\
& \left(g_{0}, \ldots, g_{i}^{1} g_{i+1}^{(1)} g_{(i+2)}^{(1)}, \ldots g_{p} \mid \sigma\left(g_{i}^{(2)}, g_{i+1}^{(2)} g_{i+2}^{(2)}\right) \sigma\left(g_{i+1}^{(3)}, g_{i+2}^{(3)}\right) a_{0} \ldots, a_{q}\right),
\end{aligned}
$$

that by using the identity () is equal to

$$
\begin{aligned}
& \left(g_{0}, \ldots, g_{i}^{1} g_{i+1}^{(1)} g_{(i+2)}^{(1)}, \ldots g_{p} \mid \sigma\left(g_{i}^{(2)}, g_{i+1}^{(2)}\right) \sigma\left(g_{i}^{(3)} g_{i+1}^{(3)}, g_{i+2}^{(3)}\right) a_{0} \ldots, a_{q}\right)= \\
& d_{i} d_{i}\left(g_{0}, \ldots, g_{p} \mid a_{0}, \ldots, a_{q}\right) .
\end{aligned}
$$

Next we check the cylindrical module condition (11). We have :

$$
\begin{aligned}
& t^{p+1} \tau^{q+1}\left(g_{0}, \ldots, g_{p} \mid a_{0}, \ldots, a_{q}\right) \\
& =t^{p+1} \tau^{q}\left(g_{0}^{(1)}, \ldots, g_{p}^{(1)} \mid S\left(g_{0}^{(0)} g_{1}^{(0)} \ldots g_{p}^{(0)}\right) \cdot a_{q}, a_{0}, \ldots, a_{q-1}\right) \\
& =t^{p+1}\left(g_{0}^{(q+1)}, \ldots, g_{p}^{(q+1)} \mid S\left(g_{0}^{(q)} \ldots g_{p}^{(q)}\right) \cdot a_{0}, S\left(g_{0}^{(q-1)} \ldots g_{p}^{(q-1)}\right) \cdot a_{1},\right. \\
& \left.\ldots, S\left(g_{0}^{(0)} \ldots g_{p}^{(0)}\right) \cdot a_{q}\right) \\
& =t^{p}\left(g_{p}^{(2 q+2)}, g_{0}^{(q+1)}, \ldots, g_{p-1}^{(q+1)} \mid\left(g_{p}^{(q+1)} S\left(g_{0}^{(q)} \ldots g_{p}^{(q)}\right)\right) \cdot a_{0},\right. \\
& \quad\left(g_{p}^{(q+2)} S\left(g_{0}^{(q-1)} \ldots g_{p}^{(q-1)}\right) \cdot a_{1}, \ldots,\left(g^{(2 q+1)} S\left(g_{0}^{(0)} \ldots g_{p}^{(0)}\right)\right) \cdot a_{q}\right) \\
& =t^{p}\left(g_{p}^{(2 q)}, g_{0}^{(q+1)}, \ldots, g_{p-1}^{(q+1)} \mid\left(S\left(g_{0}^{(q)} \ldots g_{p-1}^{(q)}\right)\right) \cdot a_{0},\right. \\
& \quad\left(g_{p}^{(q)} S\left(g_{0}^{(q-1)} \ldots g_{p}^{(q-1)}\right) \cdot a_{1}, \ldots,\left(g_{p}^{(2 q-1)} S\left(g_{0}^{(0)} \ldots g_{p}^{(0)}\right)\right) \cdot a_{q}\right) \\
& =t^{p}\left(g_{p}, g_{0}^{(q+1)}, \ldots, g_{p-1}^{(q+1)} \mid\left(S\left(g_{0}^{(q)} \ldots g_{p-1}^{(q)}\right)\right) \cdot a_{0},\right. \\
& \quad\left(S\left(g_{0}^{(q-1)} \ldots g_{p-1}^{(q-1)}\right) \cdot a_{1}, \ldots,\left(S\left(g_{0}^{(0)} \ldots g_{p-1}^{(0)}\right)\right) \cdot a_{q}\right) \\
& =\left(g_{0}, \ldots, g_{p} \mid a_{0}, \ldots, a_{q}\right) .
\end{aligned}
$$

The theorem is proved.

Next we show that the diagonal of the above cylindrical module, $d\left(A \natural_{\sigma} \mathcal{H}\right)$, is isomorphic with the cyclic module $\left(A \#_{\sigma} \mathcal{H}\right)^{\natural}$ associated with the crossed product algebra. To this end we define maps $\Phi:\left(A \#_{\sigma} \mathcal{H}\right)^{\natural} \rightarrow d\left(A \natural_{\sigma} \mathcal{H}\right)$ and 
$\Psi: d\left(A_{\natural_{\sigma}} \mathcal{H}\right) \rightarrow\left(A \#{ }_{\sigma} \mathcal{H}\right)^{\natural}$ by the following formulas

$\Phi\left(a_{0} \otimes g_{0}, \ldots, a_{n} \otimes g_{n}\right)=$

$$
\begin{gathered}
\left(g_{0}^{(2)}, g_{1}^{(3)}, \ldots, g_{n}^{(n+2)} \mid S\left(g_{0}^{(1)} g_{1}^{(2)} \ldots g_{n}^{(n+1)}\right) \cdot a_{0}, S\left(g_{1}^{(1)} g_{2}^{(2)} \ldots g_{n}^{(n)}\right) \cdot a_{1}, \ldots\right. \\
\left., S\left(g_{n-1}^{(1)} g_{n}^{(2)}\right) \cdot a_{n-1}, S\left(g_{n}^{(1)}\right) \cdot a_{n}\right),
\end{gathered}
$$

$\Psi\left(g_{0}, \ldots, g_{n} \mid a_{0}, \ldots, a_{n}\right)=$

$\left(\left(g_{0}^{(1)} g_{1}^{(1)} \ldots g_{n}^{(1)}\right) \cdot a_{0} \otimes g_{0}^{(2)},\left(g_{1}^{(2)} \ldots g_{n}^{(2)}\right) \cdot a_{1} \otimes g_{1}^{(3)}, \ldots, g_{n}^{(n+1)} \cdot a_{n} \otimes g_{n}^{(n+2)}\right)$.

Theorem 3.2. The above maps, $\Phi, \Psi$, are morphisms of cyclic modules and are inverse to one another.

Proof. It is not hard to see that $\Phi$ and $\Psi$ are inverse of each other. We just prove that $\Phi$ is a cyclic map. We first verify the commutativity of $\Phi$ and the cyclic operators, i.e., the relation $t \tau \Phi=\Phi \tau_{A \#_{\sigma} \mathcal{H}}$. We have:

$$
\begin{aligned}
& (t \tau) \Phi\left(a_{0} \otimes g_{0}, \ldots, a_{n} \otimes g_{n}\right) \\
& =t \tau\left(g_{0}^{(2)}, g_{1}^{(3)}, \ldots, g_{n}^{(n+2)} \mid S\left(g_{0}^{(1)} g_{1}^{(2)} \ldots g_{n}^{(n+1)}\right) \cdot a_{0}, S\left(g_{1}^{(1)} g_{2}^{(2)} \ldots g_{n}^{(n)}\right) \cdot a_{1}\right. \text {, } \\
& \left.\ldots, S\left(g_{n-1}^{(1)} g_{n}^{(2)}\right) \cdot a_{n-1}, S\left(g_{n}^{(1)}\right) \cdot a_{n}\right) \\
& =t\left(g_{0}^{(3)}, g_{1}^{(4)}, \ldots, g_{n}^{(n+3)} \mid S\left(g_{0}^{(2)} g_{1}^{(3)} \ldots g_{n}^{(n+2)}\right) S\left(g_{n}^{(1)}\right) \cdot a_{n}\right. \text {, } \\
& \left.S\left(g_{0}^{(1)} g_{1}^{(2)} \ldots g_{n}^{(n+1)}\right) \cdot a_{0} \ldots, S\left(g_{n-1}^{(1)} g_{n}^{(2)}\right) \cdot a_{n-1}\right) \\
& =\left(g_{n}^{((2 n+4)}, g_{0}^{(3)}, g_{1}^{(4)}, \ldots, g_{n-1}^{(n+2)} \mid g_{n}^{(n+3)} S\left(g_{0}^{(2)} g_{1}^{(3)} \ldots g_{n}^{(n+1)}\right) S\left(g_{n}^{(1)}\right) \cdot a_{n},\right. \\
& \left.g_{n}^{(n+4)} S\left(g_{0}^{(1)} g_{1}^{(2)} \ldots g_{n}^{(n+1)}\right) \cdot a_{0} \ldots, g_{n}^{(2 n+3)} S\left(g_{n-1}^{(1)} g_{n}^{(2)}\right) \cdot a_{n-1}\right) \\
& =\left(g_{n}^{((2 n+3)}, g_{0}^{(3)}, g_{1}^{(4)}, \ldots, g_{n-1}^{(n+2)} \mid \epsilon\left(g_{n}^{(n+2)}\right) S\left(g_{n}^{(1)} g_{0}^{(2)} \ldots g_{n-1}^{(n+1)}\right) \cdot a_{n},\right. \\
& \left.g_{n}^{(n+3)} S\left(g_{0}^{(1)} g_{1}^{(2)} \ldots g_{n}^{(n+1)}\right) \cdot a_{0}, \ldots, g_{n}^{(2 n+2)} S\left(g_{n-1}^{(1)} g_{n}^{(2)}\right) \cdot a_{n-1}\right) \\
& =\left(g_{n}^{((2 n+2)}, g_{0}^{(3)}, g_{1}^{(4)}, \ldots, g_{n-1}^{(n+2)} \mid S\left(g_{n}^{(1)} g_{0}^{(2)} \ldots g_{n-1}^{(n+1)}\right) \cdot a_{n}\right. \text {, } \\
& \left.\epsilon\left(g_{n}^{(n+1)}\right) S\left(g_{0}^{(1)} g_{1}^{(2)} \ldots g_{n-1}^{(n)}\right) \cdot a_{0}, \ldots, g_{n}^{(2 n)} S\left(g_{n-1}^{(1)} g_{n}^{(2)}\right) \cdot a_{n-1}\right) \\
& =\left(g_{n}^{(2)}, g_{0}^{(3)}, g_{1}^{(4)}, \ldots, g_{n-1}^{(n+2)} \mid S\left(g_{n}^{(1)} g_{0}^{(2)} \ldots g_{n-1}^{(n+1)}\right) \cdot a_{n}\right. \text {, } \\
& \left.S\left(g_{0}^{(1)} g_{1}^{(2)} \ldots g_{n-1}^{(n)}\right) \cdot a_{0}, \ldots, \ldots, \epsilon\left(g_{n}^{(2)}\right) S\left(g_{n-1}^{(1)}\right) \cdot a_{n-1}\right) \\
& =\left(g_{n}^{(2)}, g_{0}^{(3)}, \ldots, g_{n-1}^{(n+2)} \mid S\left(g_{n}^{(1)} g_{0}^{(2)} \ldots g_{n-1}^{(n+1)}\right) \cdot a_{n}\right. \text {, } \\
& \left.S\left(g_{0}^{(1)} g_{1}^{(2)} \ldots g_{n-1}^{(n)}\right) \cdot a_{0}, \ldots, \ldots, S\left(g_{n-1}^{(1)}\right) \cdot a_{n-1}\right) \\
& =\Phi\left(a_{n} \otimes g_{n}, a_{0} \otimes g_{0}, \ldots, a_{n-1} \otimes g_{n-1}\right)=\Phi\left(\tau_{A \#_{\sigma} \mathcal{H}}\left(a_{0} \otimes g_{0}, \ldots, a_{n} \otimes g_{n}\right)\right) \text {. }
\end{aligned}
$$


Next we check the commutativity of $\Phi$ and the face operators, i.e. the relation $d_{i} \delta_{i} \Phi=\Phi d_{i}^{A \#_{\sigma} \mathcal{H}}$. For $0 \leq i<n$, we have:

$$
\begin{aligned}
& d_{i} \delta_{i} \Phi\left(a_{0} \otimes g_{0}, \ldots, a_{n} \otimes g_{n}\right)= \\
& d_{i} \delta_{i}\left(g_{0}^{(2)}, g_{1}^{(3)}, \ldots, g_{n}^{(n+2)} \mid S\left(g_{0}^{(1)} g_{1}^{(2)} \ldots g_{n}^{(n+1)}\right) \cdot a_{0}, S\left(g_{1}^{(1)} g_{2}^{(2)} \ldots g_{n}^{(n)}\right) \cdot a_{1},\right. \\
& \left.\ldots, S\left(g_{n-1}^{(1)} g_{n}^{(2)}\right) \cdot a_{n-1}, S\left(g_{n}^{(1)}\right) \cdot a_{n}\right)= \\
& d_{i}\left(\left(g_{0}^{(2)}, g_{1}^{(3)}, \ldots, g_{n}^{(n+2)} \mid S\left(g_{0}^{(1)} g_{1}^{(2)} \ldots g_{n}^{(n+1)}\right) \cdot a_{0}, S\left(g_{1}^{(1)} g_{2}^{(2)} \ldots g_{n}^{(n)}\right) \cdot a_{1},\right.\right. \\
& \left.\left.\ldots, S\left(g_{i+1}^{(1)}, \ldots, g_{n}^{(n+1-i)}\right) \cdot\left(S\left(g_{i}^{(1)}\right)\left(a_{i}\right) a_{i+1}\right), \ldots, S\left(g_{n-1}^{(1)} g_{n}^{(2)}\right) \cdot a_{n-1}, S\left(g_{n}^{(1)}\right) \cdot a_{n}\right)\right)= \\
& \left(\left(g_{0}^{(2)}, g_{1}^{(3)}, \ldots, g_{i}^{(i+2)} g_{i+1}^{(i+3)}, \ldots g_{n}^{(n+2)} \mid \sigma\left(g_{i}^{(i+3)}, g_{i+1}^{(i+4)}\right) S\left(g_{0}^{(1)} g_{1}^{(2)} \ldots g_{n}^{(n+1)}\right) \cdot a_{0},\right.\right. \\
& S\left(g_{1}^{(1)} g_{2}^{(2)} \ldots g_{n}^{(n)}\right) \cdot a_{1}, \ldots, S\left(g_{i+1}^{(1)} \ldots g_{n}^{(n+1-i)}\right)\left(S\left(g_{i}^{(1)}\right)\left(a_{i}\right) a_{i+1}\right), \ldots, \\
& \left.\left.S\left(g_{n-1}^{(1)} g_{n}^{(2)}\right) \cdot a_{n-1}, S\left(g_{n}^{(1)}\right) \cdot a_{n}\right)\right)=\Phi d_{i}^{A \# \sigma \mathcal{H}}\left(a_{0} \otimes g_{0}, \ldots, a_{n} \otimes g_{n}\right) .
\end{aligned}
$$

For $i=n$, we have:

$$
\begin{aligned}
& d_{n} \delta_{n} \Phi\left(a_{0} \otimes g_{0}, \ldots, a_{n} \otimes g_{n}\right) \\
& =d_{n} \delta_{n}\left(g_{0}^{(2)}, g_{1}^{(3)}, \ldots, g_{n}^{(n+2)} \mid S\left(g_{0}^{(1)} g_{1}^{(2)} \ldots g_{n}^{(n+1)}\right) \cdot a_{0}, S\left(g_{1}^{(1)} g_{2}^{(2)} \ldots g_{n}^{(n)}\right) \cdot a_{1},\right. \\
& \left.\ldots, S\left(g_{n-1}^{(1)} g_{n}^{(2)}\right) \cdot a_{n-1}, S\left(g_{n}^{(1)}\right) \cdot a_{n}\right) \\
& =d_{n}\left(g_{0}^{(3)}, g_{1}^{(4)}, \ldots, g_{n}^{(n+3)} \mid\right. \\
& \quad\left(S\left(g_{0}^{(2)} g_{1}^{(3)} \ldots g_{n}^{(n+2)}\right) S\left(g_{n}^{(1)}\right) \cdot a_{n}\right)\left(S\left(g_{0}^{(1)} g_{1}^{(2)} \ldots g_{n}^{(n+1)}\right) \cdot a_{0}\right), \\
& \left.\quad S\left(g_{1}^{(1)} g_{2}^{(2)} \ldots g_{n}^{(n)}\right) \cdot a_{1}, \ldots, S\left(g_{n-1}^{(1)} g_{n}^{(2)}\right) \cdot a_{n-1}\right) \\
& =\left(g_{n}^{(2 n+4)} g_{0}^{(3)}, g_{1}^{(4)}, \ldots, g_{n-1}^{(n+2)} \mid\right. \\
& \quad \sigma\left(g_{n}^{(2 n+5)}, g_{0}^{(4)}\right) g_{n}^{(n+3)} \cdot\left(\left(S\left(g_{0}^{(2)} g_{1}^{(3)} \ldots g_{n}^{(n+2)}\right) S\left(g_{n}^{(1)}\right) \cdot a_{n}\right)\right. \\
& \left.\left.\left(S\left(g_{0}^{(1)} g_{1}^{(2)} \ldots g_{n}^{(n+1)}\right) \cdot a_{0}\right)\right), S\left(g_{1}^{(1)} g_{2}^{(2)} \ldots g_{n}^{(n)}\right) \cdot a_{1}, \ldots, S\left(g_{n-1}^{(1)} g_{n}^{(2)}\right) \cdot a_{n-1}\right) \\
& =\Phi d_{n}^{A \# \sigma \mathcal{H}}\left(a_{0} \otimes g_{0}, \ldots, a_{n} \otimes g_{n}\right) .
\end{aligned}
$$

The commutativity of $\Phi$ and the degeneracies are easier to check and is left to the reader. The theorem is proved.

Let $H_{\sigma}=k \# \mathcal{H}$ denote the crossed product of $\mathcal{H}$ and $k$ where $\mathcal{H}$ acts on $k$ via the counit ${ }^{\sigma} \epsilon$. One can check that the $q$-th row of the cylindrical module $A \curvearrowleft \mathcal{H}$ is the standard Hochschild complex of the algebra $\mathcal{H}_{\sigma}$ with coefficients 
in the bimodule $M_{q}=\mathcal{H} \otimes A^{\otimes(q+1)}$. Here $H_{\sigma}$ acts on $M_{q}$ on the left and right by

$$
\begin{aligned}
h \cdot\left(g \otimes a_{0} \otimes \ldots \otimes a_{q}\right) & =\sigma\left(h^{(q+3)}, g^{(2)}\right) h^{(q+2)} g^{(1)} \otimes h^{(1)} a_{0} \otimes \ldots \otimes h^{(q+1)} a_{q} \\
\left(g \otimes a_{0} \otimes \ldots \otimes a_{q}\right) \cdot h & =\sigma\left(g^{(2)}, h^{(2)}\right) g^{(1)} h^{(1)} \otimes a_{0} \otimes \ldots \otimes a_{q} .
\end{aligned}
$$

For the proof of Theorem 3.4 we need an extension of Mac Lane's isomorphism, which relate group homology to Hochschild homology, to Hopf algebras.

We recall that the Hopf homology of a Hopf algebra $\mathcal{H}$ with coefficients in a left $\mathcal{H}$-module $M$ is the homology of the following complex

$$
M \stackrel{d_{0}}{\leftarrow} \mathcal{H} \otimes M \stackrel{d_{1}}{\leftarrow} \mathcal{H} \otimes \mathcal{H} \otimes M \stackrel{d_{2}}{\leftarrow} \ldots \mathcal{H}^{\otimes n} \otimes M \stackrel{d_{n}}{\leftarrow} \mathcal{H}^{\otimes(n+1)} \otimes M \leftarrow \ldots,
$$

where the differential $d_{n}$ is given by

$$
\begin{aligned}
& d_{n}\left(h_{0} \otimes h_{1} \otimes \ldots \otimes h_{n} \otimes m\right)=\epsilon\left(h_{0}\right) h_{1} \otimes \ldots \otimes h_{n} \otimes m+ \\
& \sum_{1 \leq i \leq n-1}(-1)^{i} h_{0} \otimes \ldots \otimes h_{i} h_{i+1} \otimes \ldots \otimes h_{n} \otimes m+(-1)^{n} h_{0} \otimes h_{1} \otimes \ldots \otimes h_{n-1} \otimes h m .
\end{aligned}
$$

We denote the $n$th Hopf homology group of $\mathcal{H}$ with coefficients in $M$ by $H_{n}(\mathcal{H} ; M)$.

Let $M$ be an $\mathcal{H}_{\sigma}$-bimodule. We can convert $M$ to a new left $\mathcal{H}$-module, $\widetilde{M}=M$, where the action of $\mathcal{H}$ on $\widetilde{M}$ is defined by

$$
h>m=\sigma^{-1}\left(S\left(h^{(2)}\right), h^{(3)}\right) \overline{h^{(4)}} m \overline{S\left(h^{(1)}\right)},
$$

where $\bar{h}$ denotes the image of $h$ in $\mathcal{H}_{\sigma}$ under the map $h \rightarrow 1 \# h$.

The following result was first proved in [9] for $\sigma$ a trivial cocycle.

Theorem 3.3. ( Mac Lane Isomorphism for Hopf crossed product) Let $M$ be an $\mathcal{H}_{\sigma}$-bimodule and $\widetilde{M}$ be defined as above. Then the following map defines an isomorphism between Hochschild and Hopf homology complexes:

$$
\begin{gathered}
\Theta: C_{n}\left(\mathcal{H}_{\sigma}, M\right) \longrightarrow C_{n}(\mathcal{H} ; \widetilde{M}) \\
\Theta\left(\bar{h}_{1} \otimes \ldots \otimes \bar{h}_{n} \otimes m\right)=h_{1}^{(2)} \otimes h_{2}^{(2)} \otimes \ldots \otimes h_{n}^{(2)} \otimes m \overline{h_{1}^{(1)}} \ldots \overline{h_{n}^{(1)}} .
\end{gathered}
$$

Proof. We show more than what we need for the proof, namely we show that $\Theta$ is an isomorphisms of of simplicial modules. We have:

$$
\begin{aligned}
& \Theta \delta_{0}\left(\bar{h}_{1} \otimes \ldots \otimes \bar{h}_{n} \otimes m\right)=\Theta\left(\bar{h}_{2} \otimes \ldots \otimes \bar{h}_{n} \otimes m \bar{h}_{1}\right)= \\
& h_{2}^{(2)} \otimes \ldots \otimes h_{n}^{(2)} \otimes m \overline{h_{1}} \overline{h_{n}^{(2)}} \ldots \overline{h_{n}^{(1)}}=\delta_{0} \Theta\left(\bar{h}_{1} \otimes \ldots \otimes \bar{h}_{n} \otimes m\right) .
\end{aligned}
$$


For $0 \leq i \leq n$, we have

$$
\begin{aligned}
& \Theta \delta_{i}\left(\bar{h}_{1} \otimes \ldots \otimes \bar{h}_{n} \otimes m\right)= \\
& \Theta\left(\bar{h}_{1} \otimes \ldots \otimes\left(\overline{h_{i}}\right)\left(\overline{h_{i+1}}\right) \otimes \ldots \otimes \bar{h}_{n} \otimes m\right)= \\
& h_{1}^{(2)} \otimes h_{2}^{(2)} \otimes \ldots \otimes h_{i}^{(2)} h_{i+1}^{(2)} \otimes \ldots \otimes h_{n}^{(2)} \otimes m \overline{h_{1}^{(1)}} \ldots \overline{h_{n}^{(1)}}= \\
& \delta_{i} \Theta\left(\bar{h}_{1} \otimes \ldots \otimes \bar{h}_{n} \otimes m\right) .
\end{aligned}
$$

We leave it to the reader to check the commutativity of $\Theta$ with the last face and the degeneracies.

To finish the proof one can check that the following map is the inverse of $\Theta$

$$
\begin{gathered}
\mathfrak{T}: C_{n}(\mathcal{H} ; \widetilde{M}) \longrightarrow C_{n}\left(\mathcal{H}_{\sigma}, M\right) \\
\mathfrak{T}\left(h_{1} \otimes \ldots \otimes h_{n} \otimes m\right)= \\
\sigma^{-1}\left(S\left(h_{1}^{(2)}\right), h_{1}^{(3)}\right) \ldots \sigma^{-1}\left(S\left(h_{n}^{(2)}\right), h_{n}^{(3)}\right) \overline{h_{1}^{(4)}} \otimes \ldots \otimes \overline{h_{n}^{(4)}} \otimes m \overline{S\left(h_{n}^{(1)}\right)} \ldots \overline{S\left(h_{1}^{(1)}\right)} .
\end{gathered}
$$

We apply the generalized cyclic Eilenberg-Zilber theorem (Theorem 2.1) to the cylindrical module $A \natural_{\sigma} \mathcal{H}$ to derive a spectral sequence for the cyclic homology of $A \#_{\sigma} \mathcal{H}$. We have

$$
\operatorname{Tot}\left(A \natural_{\sigma} \mathcal{H}\right) \rightarrow d\left(A \natural_{\sigma} \mathcal{H}\right) \cong\left(A \#_{\sigma} \mathcal{H}\right)^{\natural},
$$

where the first map is a quasi-isomorphism of mixed complexes given in Theorem 2.1 and the second map is the isomorphism given in Theorem 3.2. We filter the mixed complex $\operatorname{Tot}\left(A \natural_{\sigma} \mathcal{H}\right)$ by sub mixed complexes

$$
F^{i}\left(\operatorname{Tot}\left(A \natural_{\sigma} \mathcal{H}\right)\right)_{n}=\bigoplus_{\substack{p+q=n \\ q \leq i}} A^{\otimes(p+1)} \otimes \mathcal{H}^{\otimes(q+1)} .
$$

This gives us an spectral sequence that converges to $H C \cdot\left(A \#_{\sigma} \mathcal{H}\right)$. We can then apply Theorem 3.3 to identify the $E^{1}$-term of this spectral sequence, i.e. the homology of rows, as Hopf homologies of $\mathcal{H}$, with coefficients in $M_{q}=\mathcal{H} \otimes A^{\otimes(q+1)}$, where $\mathcal{H}$ acts on $M_{q}$ by

$h$ マ $\left(g \otimes a_{0} \otimes a_{1} \otimes \ldots \otimes a_{q}\right)=\sigma^{-1}\left(S\left(h^{(3)}\right), h^{(4)}\right) \sigma\left(h^{(q+6)}, g^{(1)}\right) \sigma\left(h^{(q+7)} g^{(2)}, S\left(h^{2}\right)\right)$ $h^{(q+8)} g^{(3)} S\left(h^{(1)}\right) \otimes h^{(5)}\left(a_{0}\right) \otimes \ldots \otimes h^{(q+5)}\left(a_{q}\right)$.

This proves the following theorem. 
Theorem 3.4. There is a spectral sequence that converges to $H C_{p+q}\left(A \#_{\sigma} \mathcal{H}\right)$. The $E^{1}$-term of this spectral sequence is given by

$$
E_{p, q}^{1}=H_{p}\left(\mathcal{H} ; M_{q}\right) .
$$

Given any cylindrical module $X=\left\{X_{p, q}\right\}_{p, q \geq 0}$, if we compute the Hochschild homologies of rows of $X$ we obtain a new bigraded $k$-module $X^{\prime}=\left\{X_{p, q}^{\prime}\right\}_{p, q \geq 0}$. We clime that the columns of $X^{\prime}$, i.e. $\left\{X_{p, q}^{\prime}\right\}_{q \geq 0}$ form a cyclic module for each $p \geq 0$. For some especial cases one can find the proof in [7, 1]. The same proof, however, works in the general case. This observation proves the following proposition.

Proposition 3.5. The $p^{\text {th }}$ column of $E^{1}$, i.e. $\left\{H_{p}\left(\mathcal{H} ; M_{q}\right)\right\}_{q \geq 0}$ is a cyclic module for each $p \geq 0$.

We denote the $p^{\text {th }}$ column of $E^{1}$ by $N_{p}$. One can observe that the induced differential $d^{1}$ on $E^{1}$ is simply the differential $b+B$ associated to the cyclic modules $N_{p}$. This finishes the proof of the following theorem.

Theorem 3.6. The $E^{2}$ term of the spectral sequence in Theorem 3.4 is

$$
E_{p, q}^{2}=H C_{q}\left(N_{p}\right) .
$$

\section{References}

[1] R. Akbarpour, and M. Khalkhali, Hopf Algebra Equivariant Cyclic Homology and Cyclic Homology of Crossed Product Algebras. arXiv: math.KT/0011248, To appear in Crelle's Journal.

[2] R. J. Blattner, M. Cohen, and S. Montgomery, Crossed products and inner action of Hopf algebras. Trans. Amer. Math. Soc. 298 (1986), 671711.

[3] A. Connes, Noncommutative geometry. Academic Press, Inc., San Diego, CA, 1994.

[4] A. Connes, Cohomologie cyclique et foncteurs Ext ${ }^{n}$. (French) (Cyclic cohomology and functors Ext $^{n}$ ) C. R. Acad. Sci. Paris Sr. I Math. 296 (1983), no. 23, 953-958. 
[5] Y. Doi, and M. Takeuchi, Cleft comodule algebras by bialgebra, Comm. in Alg. 14 (1986), 801-817.

[6] B. Fĕgin, and B. L. Tsygan, Additive $K$-theory. $K$-theory, arithmetic and geometry (Moscow, 1984-1986), 67-209, Lecture Notes in Math., 1289, Springer, Berlin, 1987.

[7] E. Getzler, and J. D. S Jones, The cyclic homology of crossed product algebras. J. Reine Angew. Math. 445 (1993), 161-174.

[8] J. A. Guccione, and J. J. Guccione, Hochschild (co)homology of Hopf crossed products. math.KT/0104075.

[9] M. Khalkhali, and B. Rangipour, A New Cyclic Module for Hopf Algebras, K-Theory 27, 111-131, Nov. 2002.

[10] M. Khalkhali, and B. Rangipour, On the Generalized Cyclic EilenbergZilber Theorem, to appear in Canadian Math. Bulletin.

[11] J. L. Loday, Cyclic Homology. Springer-Verlag, (1992).

[12] V. Nistor, Group homology and the cyclic homology of crossed products, Invent. Math. 99, (1989) 411-424.

[13] M. A. Rieffel, Morita equivalence for $C^{*}$-algebras and $W^{*}$-algebras, J. pure Apll. Algebras 5 (1974), 51-96.

[14] M. Sweedler, Hopf algebras. Mathematics Lecture Note Series W. A. Benjamin, Inc., New York 1969. 